\title{
REVIEW \\ Nutrition and growth in children with cerebral palsy: setting the scene
}

\author{
PB Sullivan \\ European Journal of Clinical Nutrition (2013) 67, S3-S4; doi:10.1038/ejcn.2013.222
}

Keywords: enteral nutrition; cerebral palsy; undernutrition; feeding difficulties; gastrostomy

The early text books on cerebral palsy in children made little or no mention of the feeding and nutritional problems that are common in this group of children. Even today the nutrition and growth deficits in this group of children are often under recognised or considered to be of low priority. It is really only in the last couple of decades that the nutritional needs of children with cerebral palsy have been the subject of research interest. It is also important to note that neurological impairment in children is here to stay. Epidemiological data on the prevalence of cerebral palsy show that in those who are premature, weighing $<1500 \mathrm{~g}$ at birth, cerebral palsy occurs in up to 65 per 1000 live births. ${ }^{1}$ The recent EPICURE 2 study showed that although the survival of preterm neonates of 22-25 weeks gestation has improved from 1995 to 2006 , the proportion with severe disability is unchanged. ${ }^{2}$ Moreover, the life expectancy of these children is gradually improving, with survival curves for 10-year old boys with cerebral palsy showing a $50 \%$ survival into their late 20 s (http://www.lifeexpectancy.org/survival.shtml). The prevalence and consequences of feeding difficulties in individuals with cerebral palsy therefore should not be underestimated. Reilly et al. ${ }^{3}$ showed feeding difficulties in up to $85 \%$ of children with spastic quadriplegia, and the North American Growth and Cerebral Palsy Project showed that two-thirds of such children were stunted and $44 \%$ had low fat stores. These growth deficits were found to correlate with the severity of cerebral palsy. ${ }^{4}$

The causes of the feeding problems in children with cerebral palsy are multiple. A significant reduction in nutritional intake comes primarily from oropharyngeal incoordination, which itself is associated with slow rates of feeding, prolonged feeding times, excessive spillage of food and compromise to the safety of the swallow. In addition to oropharyngeal incoordination, vomiting, poor dentition, early satiety, communication defects and behavioural disturbances all contribute to the undernutrition, which is characteristic of many children with severe cerebral palsy.

The adverse consequences of such undernutrition must be appreciated. The main consequence of undernutrition is, of course, growth failure. Other consequences include decreased cerebral function, which is associated with reduced potential for development, reduced responsiveness and noticeable withdrawal and irritability. In addition, impaired immune function, which increases the risk of infection, reduced circulation time, which increases the risk of poor healing and pressure sores, and diminished respiratory muscle strength, which is associated with weak cough and more chest infections, are all corollaries of undernutrition. As argued above, the number of these children will not decline and their life expectancy will increase; therefore, attention to their nutritional status is an indispensable part of the overall management of their health.

Assessment of feeding and nutrition in children with cerebral palsy should focus on three key questions: ${ }^{5}$

1. Is feeding adequate? Is it meeting the nutritional needs of the child for normal physiological function and optimum growth?

2. Is feeding safe? Does the degree of oromotor impairment compromise the safety of the airway so that the child is at risk of clinically significant aspiration?

3. Is feeding efficient, comfortable and enjoyable? Feeding is a social function as well as a nutritional imperative and can present a major challenge to both the child and the parent. Some mothers spend many hours a day trying to feed their disabled child and there is no doubt that quality of life in parents of children with cerebral palsy is significantly impaired. ${ }^{6}$

A multidisciplinary team approach to assessment is required and this may require involvement from a neurodisability pediatrician, pediatric gastroenterologist, pediatric dietician, clinical nurse specialist, speech and language therapist, and occupational and physiotherapists. Clinical assessment should embrace not only the usual medical history but also feeding and dietetic history. An examination should extend beyond routine clinical examination to a detailed anthropometric analysis of nutritional status. Additional investigations such as videofluoroscopy and prolonged lower oesophageal $\mathrm{pH}$ monitoring may be required. It is important to recognize that children with cerebral palsy have a significant degree of foregut dysmotility and, as a consequence, gastroesophageal reflux, delayed gastric emptying and so on may complicate nutritional management.

Nutritional management seeks to optimize nutritional status by providing dietary advice and counselling and, if the nutritional needs of the child cannot be met by normal foods alone, by the use of oral nutritional supplements. The degree of oromotor function, however, may necessitate tube feeding. Although nasogastric tube feeding is useful as a short-term measure to demonstrate the ability to tolerate tube feeding and to improve nutritional status before gastrostomy feeding, it is not a long-term solution, which itself would be more appropriately provided by gastrostomy feeding.

Indications for gastrostomy feeding are the following: clinically unsafe swallow, malnutrition secondary to oromotor dysfunction and nasogastric tube dependency. It is important to appreciate, 
however, that gastrostomy is not a panacea for nutritional disorders and growth failure in cerebral palsy. The introduction of the idea of gastrostomy feeding to caregivers of disabled children requires a great deal of care and sensitivity. There is no doubt that the quality of life of caregivers, particularly where feeding times are prolonged, is likely to be improved by this intervention. $^{6}$ Nevertheless, gastrostomy tube feeding is not without its complications; it is a surgical procedure and may be associated with peritonitis or secondary gastroesophageal reflux, and minor issues such as skin excoriation and granulation are not uncommon. One of the reasons that deter doctors from inserting gastrostomy feeds in these children is the fear of overfeeding. This is a realistic concern and certainly with modern high calorie $(1.0-1.5 \mathrm{kcal} / \mathrm{ml})$ enteral tube feeds, there is a risk of overfeeding these children who have a reduced total energy expenditure compared with ambulant children. ${ }^{7}$ The key thing is to seek to optimize nutritional status so that neither undernutrition nor overnutrition occurs. This can be achieved through adequate monitoring of nutritional status and feeding regimens.

Adequate enteral feeding leads to increases in weight, weight for height, muscle mass, improvements in peripheral circulation, immune function, cognitive ability and general well-being for the disabled child. It is important to emphasise the requirement for follow-up, which would include monitoring of growth and of development of symptoms suggestive of secondary gastroesophageal reflux.

There is a need for guidelines for the screening and nutritional management of children with cerebral palsy for non-experts. Guidelines on screening should include what, when, how and by whom. Guidelines on assessment should describe what should be done, when, how and by whom. Nutritional management guidelines should cover what intervention should be used, when, by whom and should provide a plan for follow-up. These guidelines should also consider psychological elements.
This supplement is provided as a professional service by the Paediatric Division of Nutricia Advanced Medical Nutrition.

\section{CONFLICT OF INTEREST}

PBS has received consulting fees from Danone Limited, lecture fees from Nestec Limited and Nutricia Limited, and grant support from Nutricia, the Castang Foundation and Sparks.

\section{REFERENCES}

1 Winter S, Autry A, Boyle C, Yeargin-Allsopp M. Trends in the prevalence of cerebral palsy in a population-based study. Pediatrics 2002; 110 1220-1225.

2 Costeloe KL, Hennessy EM, Haider S, Stacey F, Marlow N, Draper ES. Short term outcomes after extreme preterm birth in England: comparison of two birth cohorts in 1995 and 2006 (the EPICure studies). BMJ 2012; 345: e7976.

3 Reilly S, Skuse D, Poblete X. Prevalence of feeding problems and oral motor dysfunction in children with cerebral palsy: a community survey. J Pediatr 1996; 129: 877-882.

4 Fung EB, Samson-Fang L, Stallings VA, Conaway M, Liptak G, Henderson RC et al. Feeding dysfunction is associated with poor growth and health status in children with cerebral palsy. J Am Diet Assoc 2002; 102: 3-73.

5 Couriel JM, Bisset R, Miller R, Thomas A, Clarke M. Assessment of feeding problems in neurodevelopmental handicap: a team approach. Arch Dis Child 1993; 69: 609-613.

6 Sullivan PB, Juszczak E, Bachlet AM, Thomas AG, Lambert B, Vernon-Roberts A et al. Impact of gastrostomy tube feeding on the quality of life of carers of children with cerebral palsy. Dev Med Child Neurol 2004; 46: 796-800.

7 Sullivan PB, Alder N, Bachlet AM, Grant H, Juszczak E, Henry J et al. Gastrostomy feeding in cerebral palsy: too much of a good thing? Dev Med Child Neurol 2006; 48: 877-882.

(i) $\Theta$ This work is licensed under a Creative Commons AttributionNonCommercial-NoDerivs 3.0 Unported License. To view a copy of this license, visit http://creativecommons.org/licenses/by-nc-nd/3.0/ 